\title{
Editorial \\ SELDI-TOF proteomic profiling of breast carcinomas identifies clinicopathologically relevant groups of patients similar to previously defined clusters from cDNA expression
}

\author{
Bashar A Zeidan and Paul A Townsend
}

Human Genetics Division, School of Medicine, University of Southampton, Southampton General Hospital, Tremona Road, Southampton, SO16 6YD, UK

Corresponding author: Paul A Townsend, p.a.townsend@soton.ac.uk

Published: 30 June 2008

Breast Cancer Research 2008, 10:107 (doi:10.1186/bcr2107)

This article is online at http://breast-cancer-research.com/content/10/3/107

(c) 2008 BioMed Central Ltd

See related research article by Brozokova et al., http://breast-cancer-research.com/content/10/3/R48

\begin{abstract}
Expression profiling and biomarker(s) discovery aim to provide means for tumour diagnosis, classification, therapy response and prognosis. The identification of novel markers could potentially lead to the building of robust early detection strategies and personalized, effective breast cancer therapies that would improve patient outcome. Recent evidence supports the hypothesis that genomic expression profiling using microarray analysis is a reliable method for breast cancer classification and prognostication. However, genes clearly do not act by themselves, or indeed they do not have catalytic or signalling capabilities. Hence, genetic biomarker information alone cannot perfectly predict cancer and its response to treatment. Genes clearly exert their effect after transcription through translation into active proteins. Consequently, postgenomic projects correlating protein expression profiles with tumour classification have led to some established biomarkers. In this regard, these biomarkers associate with disease prediction and can be associated with treatment response. Recently, Brozokova and colleagues demonstrated that surface-enhanced laser desorption ionization time of flight mass spectrometry (SELDI-TOF MS) profiling of breast cancer tissue proteomes can potentially expand the biomarker repertoire and our knowledge of breast cancer behaviour.
\end{abstract}

New technologies for predicting or classifying cancer are constantly evolving. Reported in this issue, a study by Brozokova and coworkers [1] demonstrates that protein expression profiling may enhance our accuracy of detection and prognostication in breast cancer. It has become clear that histological analysis is not, by itself, adequate in predicting clinical outcome and insensitive in identifying the optimal therapeutic strategy in many cancers [2]. Individual tumours often carry a specific molecular footprint that may have more in common with another tumour of different histological type, and emphasis is now shifting toward specific clinical management of these individual molecular patterns rather than a blanket, 'one size fits all' therapy for tumours with common histology. Breast cancer is a major killer that remains challenging in terms of molecular complexity and treatment response. The multifactorial nature of breast cancer lends itself to the use of multiple biomarkers for early detection, monitoring of response to therapy, and clinical outcome prediction. Microarray-based gene expression profiling has provided new insight into the molecular intricacy of breast cancer and has provided a clinically valuable breast cancer classification system [3-5]. However, cDNA profiles do not provide a complete picture. After all, it is proteins in all their many post-translational coats and not RNA transcripts - that run the intracellular machinery. Thus, there is a need to identify new, more accurate markers of disease. Enter proteomic profiling.

Brozokova and colleagues [1] employed proteomics using surface-enhanced laser desorption ionization time of flight mass spectrometry (SELDI-TOF MS) to identify relevant molecular signatures of breast cancer. This work revealed subgroups of patients with differential co-expression of protein peaks in diverse tumour subclasses, and demonstrated differing clinicopathological characteristics in tumour type, nuclear grade, hormonal status, mucin 1, and cytokeratin $5 / 6$ or 14 between these groups. Significantly, the patient subgroups identified by hierarchical clustering of SELDI-TOF MS peaks were analogous to breast cancer classifications based on gene expression profiling, classifying the tumours into luminal, basal and HER2 subtypes [3].

Use of SELDI TOF MS has three main advantages. First, the reliability of signatures of breast cancer gene expression is becoming increasingly acknowledged, and proteomic profiling is considered to be the final step in drawing a biological picture of cancers. Second, despite variable biomarker

$\mathrm{HSP}=$ heat shock protein; SELDI-TOF MS = surface-enhanced laser desorption ionization time of flight mass spectrometry. 
detection from separate studies [6-10], mass spectrometry represents an additional approach to mine novel proteins. Third, these new biomarkers may well relate to tumour development and behaviour extrapolated from minimal starting material and high throughput proteomic capabilities.

A fundamental part of the study conducted by Brozokova and colleagues was the progression from descriptive knowledge of protein peak signature classification to the identification of two proposed biomarkers. Here, they identified two significant biomarkers, namely heat shock protein (HSP) 27 and annexin $\mathrm{V}$, which were over-expressed in subcohorts of patients corresponding to luminal A subtypes. Significantly, HSP27 appears to be a bona fide biomarker, because it was shown to be elevated in oestrogen receptor positive patients in a previous study [11]. Further confirmation has been reported; specifically, HSP27 and a related protein isoform of the annexin family were found to correlate with chemoresistance in breast cancer [12].

Evidently, SELDI-TOF MS and mass spectrometry in general allow a more rapid identification of many potential biomarkers from biological samples. This study and those described above support the use of SELDI as a complementary method of breast cancer classification and biomarker discovery. The identification of biomarker proteins in this work is a key element in elucidating the relevant pathways of breast cancer, and highlights the possibility that some of these biomarkers may be monitored/targeted in future treatment modalities. There are some issues regarding SELDI's role in disease prognosis and classification, which would benefit from larger, multicentre validation studies. However, as mass spectrometry technology evolves, it is becoming increasingly clear that changes in proteomic profiles of breast cancer will aid in disease stratification and prognostication, and will significantly help to guide selection of treatment regimens.

\section{Competing interests}

The authors declare that they have no competing interests.

\section{References}

1. Brozkova K, Budinska E, Bouchal P, Hernychova L, Knoflickova D, Valik D Vyzula R, Vojtesek B, Nenutil R: Surface-enhanced laser desorption/ionization time-of-flight proteomic profiling of breast carcinomas identifies clinicopathologically relevant groups of patients similar to previously defined clusters from cDNA expression. Breast Cancer Res 2008, 10:R48.

2. Tonon G, Brennan C, Protopopov A, Maulik G, Feng B, Zhang Y, Khatry DB, You MJ, Aguirre AJ, Martin ES, Yang Z, Ji H, Chin L, Wong KK, Depinho RA: Common and contrasting genomic profiles among the major human lung cancer subtypes. Cold Spring Harb Symp Quant Biol 2005, 70:11-24.

3. Brenton JD, Carey LA, Ahmed AA, Caldas C: Molecular classification and molecular forecasting of breast cancer: ready for clinical application? J Clin Oncol 2005, 23:7350-7360.

4. Perou CM, Sørlie T, Eisen MB, van de Rijn M, Jeffrey SS, Rees CA, Pollack JR, Ross DT, Johnsen H, Akslen LA, Fluge O, Pergamenschikov A, Williams C, Zhu SX, Lønning PE, Børresen-Dale AL, Brown PO, Botstein D: Molecular portraits of human breast tumours. Nature 2000, 406:747-752.

5. Sorlie T, Perou CM, Tibshirani R, Aas T, Geisler S, Johnsen H Hastie T, Eisen MB, van de Rijn M, Jeffrey SS, Thorsen T, Quist H,
Matese JC, Brown PO, Botstein D, Eystein Lønning P, BørresenDale AL: Gene expression patterns of breast carcinomas distinguish tumor subclasses with clinical implications. Proc Natl Acad Sci USA 2001, 98:10869-10874.

6. Li J, Zhang Z, Rosenzweig J, Wang YY, Chan DW: Proteomics and bioinformatics approaches for identification of serum biomarkers to detect breast cancer. Clin Chem 2002, 48:12961304.

7. Becker S, Cazares LH, Watson P, Lynch H, Semmes OJ, Drake $\mathrm{RR}$, Laronga C: Surfaced-enhanced laser desorption/ionization time-of-flight (SELDI-TOF) differentiation of serum protein profiles of BRCA-1 and sporadic breast cancer. Ann Surg Oncol 2004, 11:907-914.

8. Li J, Zhao J, Yu X, Lange J, Kuerer $\mathrm{H}$, Krishnamurthy S Schilling $\mathrm{E}$ Khan SA, Sukumar S, Chan DW: Identification of biomarkers for breast cancer in nipple aspiration and ductal lavage fluid. Clin Cancer Res 2005, 11:8312-8320.

9. Ricolleau G, Charbonnel C, Lode L, Loussouarn D, Joalland MP, Bogumil R Jourdain S, Minvielle S, Campone M, Déporte-Fety R, Campion $L$, Jézéquel $P$ : Surface-enhanced laser desorption/ionization time of flight mass spectrometry protein profiling identifies ubiquitin and ferritin light chain as prognostic biomarkers in node-negative breast cancer tumors. Proteomics 2006, 6:1963-1975.

10. Belluco C, Petricoin EF, Mammano E, Facchiano F, Ross-Rucker S, Nitti D Di Maggio C, Liu C, Lise M, Liotta LA, Whiteley G: Serum proteomic analysis identifies a highly sensitive and specific discriminatory pattern in stage 1 breast cancer. Ann Surg Oncol 2007, 14:2470-2476.

11. Ciocca DR, Stati AO, Amprino de Castro MM: Colocalization of estrogen and progesterone receptors with an estrogen-regulated heat shock protein in paraffin sections of human breast and endometrial cancer tissue. Breast Cancer Res Treat 1990, 16:243-251.

12. Chuthapisith S, Layfield R, Kerr ID, Hughes C, Eremin O: Proteomic profiling of MCF-7 breast cancer cells with chemoresistance to different types of anti-cancer drugs. Int J Oncol 2007, 30:1545-1551. 